\title{
APLICACIONES DEL GRC EN ESPAÑA Y ARGELIA*
}

\author{
(GRC APPLICATIONS IN SPAIN AND ALGERIA)
}

Jesús Rodríguez Santiago, Ingeniero de Caminos. Dragados y Construcciones, S. A.

Miguel Jordán Reyes, Ingeniero. Dragados y Construcciones, S. A.

\section{RESUMEN}

Este artículo resume la experiencia obtenida en la utilización del mortero de cemento reforzado con fibra de vidrio (GRC) en el campo de la construcción. Se describen en primer lugar las caracteristicas del material y el proceso de fabricación en factoria de paneles de GRC. Se exponen a continuación algunas realizaciones llevadas a cabo con este material destacando, por su volumen y rapidez de ejecución, el conjunto de fachadas para edificios de viviendas, hospitales y centros escolares construidos en Argelia. También se comentan otras aplicaciones del GRC para la rehabilitación y decoración interior de edificios y para la construcción de elementos singulares. Finalmente, en el apartado de las conclusiones se menciona la necesidad de seguir investigando en el comportamiento a largo plazo de este material para poder aumentar su utilización en la construcción.

\section{SUMMARY}

This article offers a brief summary of our experience using glass fibre reinforced cement (GRC) in the construction field. Begining with a description of the material's characteristics and the process by which GRC panels are manufactured, the article goes on to discuss some of the projects carried out with this material. Special mention is made of a group of projects in Algeria where numerous facades of buildings for housing, schools and hospitals were built in record time. Other uses of GRC are also noted, such as: facade rehabilitation, interior decoration and special elements. The closing remarks emphasize the need for continued research into GRC long term behaviour to enable its increased use in the construction industry.

\section{INTRODUCCION}

Este artículo resume algunas experiencias obtenidas por la empresa española DRAGADOS y CONSTRUCCIONES S. A., en la construcción de elementos prefabricados de GRC a lo largo de los últimos diez años, destacando de manera especial un conjunto de proyectos realizados en Argelia.

La prefabricación de las piezas de GRC se lleva a cabo en dos factorias situadas en los alrededores de Madrid y Sevilla respectivamente. La primera abastece las zonas centro y norte de España mientras que la segunda suministra prefabricados a la zona del sur de España y para la exportación a través del puerto de Cádiz.

La experiencia obtenida con el uso de este material ha sido muy amplia, habiéndose realizado fachadas de distinto tipo de edificios, elementos decorativos, rehabilitación de fachadas, encofra-

(*) Este articulo corresponde a la traducción española del trabajo presentado en inglés por el primer autor al "Symposium FRC-86” celebrado en Sheffield (Julio-1986). dos permanentes de losas de hormigón, cabinas, etc. en una cantidad que superan los 600.000 metros cuadrados de GRC.

\section{FABRICACION DE ELEMENTOS DE GRC}

Para la fabricación de piezas de GRC se emplea un mortero constituido por una arena silicea de tamaño máximo $1 \mathrm{~mm}$ y un cemento Portland P-450 ARI (alta resistencia inicial) o un cemento Portland blanco P-450 B con una relación cemento/arena 1:1. La relación agua/cemento varía entre 0,30 Y 0,35 y suele añadirse un superplastificante en una proporción del $1 \%$ del peso de cemento. La mezcla se realiza en una mezcladora de eje vertical con una velocidad de rotación de 720 a 1.440 revoluciones por minuto.

EI GRC se fabrica por proyección empleando una fibra de vidrio resistente a los alcalis (CEMFIL 2) de $30-40 \mathrm{~mm}$ de longitud y con una dosificación en peso del $5 \%$ del total de la mezcla. Con este tipo de mezclas se obtienen en el ensayo de flexión valores del módulo de rotura de $20 \mathrm{MPa}$ y valores del limite de proporcionalidad del $40 \%$ de los anteriores. 


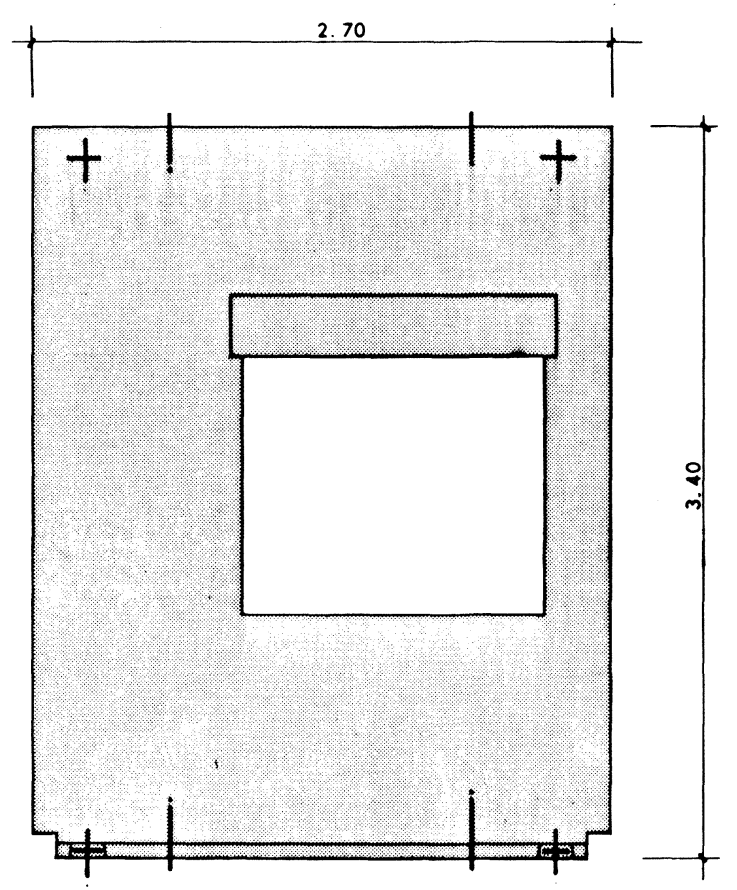

Alzado posterior
+ Puntos de anclaje del panel a la estructura del edificio.

! Puntos de fijación del sistema de elevación del panel.

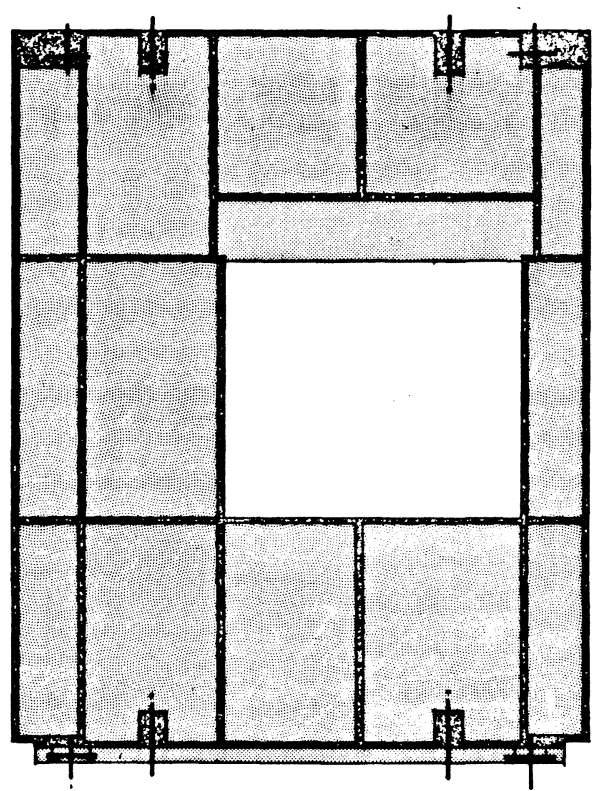

Distribución de nervios

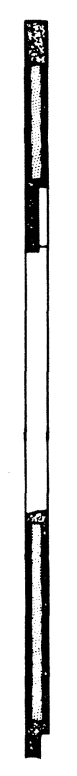

Sección

Fig. 1. - Panel sandwich de GRC.

La proyección del GRC se realiza contra unos moldes del mismo material fabricados a partir de un modelo de madera al que, si se desea conseguir un determinado dibujo de acabado, se le pega un revestimiento vinilico con tal dibujo. Las piezas se extraen de los moldes a las 24 horas acortándose dicho plazo en épocas con temperaturas ambiente elevadas. Para las operaciones de extracción e izado de las piezas se dejan embebidos en el GRC unos casquillos roscados anclados mediante patillas a los que posteriormente se les roscan los útiles para la manipulación de las piezas.

Una vez extraídas las piezas de los moldes se acopian en una zona de las naves habilitada para la operación de curado que se lleva a cabo durante un periodo de una semana en un ambiente de humedad relativa del $80 \%$ y una temperatura de 18-20 grados centígrados.

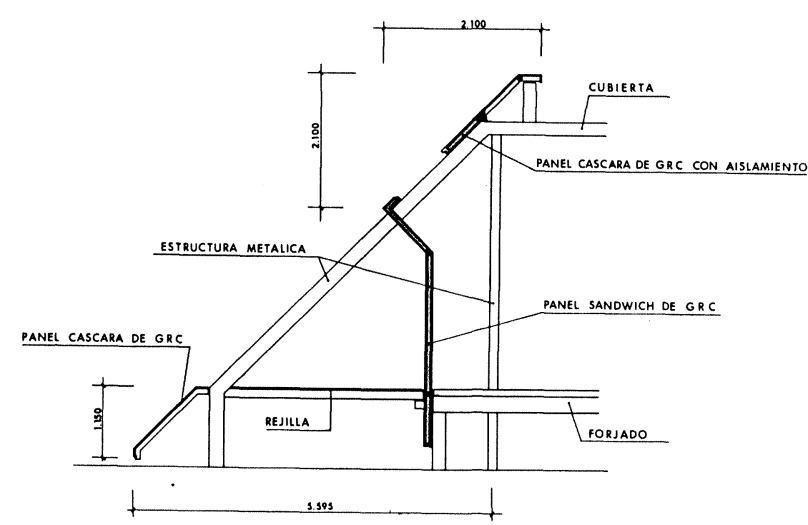

Fig. 2. - Centro para el seguimiento de satélites (Guadalajara).
Se diseñan tanto paneles cáscara como paneles sandwich con espesores de cáscara de orden de $10 \mathrm{~mm}$ (Figs. 1 y 2). En todos los casos tienen nervios de GRC de $20 \mathrm{~mm}$ de espesor y un canto acorde con el tamaño del panel y la distancia entre apoyos. El material comúnmente empleado para el núcleo de los paneles sandwich es el poliestireno expandido de $10-12 \mathrm{~kg} / \mathrm{m}^{3}$ de densidad usándose también el hormigón con perlas de poliestireno expandido.

Los paneles empleados para el cerramiento de edificios se unen a la estructura de éstos o a una estructura auxiliar mediante anclajes metálicos que permiten los posibles movimientos del panel debidos a las deformaciones impuestas por variaciones térmicas, de humedad... (Fig. 3). En los paneles se dejan embebidos unos carriles y en la estructura unas chapas metálicas a las que se sueldan unos angulares de chapa plegada que se unen a los carriles del panel mediante tornillos.

La cara exterior de los paneles se termina siempre o bien con la aplicación de una pintura de poliuretano, acrilato isocianato o similar (aprox. $300 \mathrm{gr} / \mathrm{m}^{2}$ ) o mediante un acabado pétreo. Este último se realiza mediante el vertido previo en el molde de una mezcla de mortero con un árido de determinadas caracteristicas según el acabado deseado. La aplicación de un retardador de fraguado al molde (en forma liquida o en papel) permite descubrir posteriormente el árido mediante un cepillado con agua. La cara interior de los paneles sandwich se termina embebiendo en el GRC un tejido de fibra mineral tipo Velimat, lo que permite obtener una terminación superficial 
adecuada para la aplicación de una pintura o un acabado convencional.

El tratamiento de las juntas entre los paneles no difiere sustancialmente de lo que es habitual en los prefabricados de hormigón. Suelen sellarse con cordones de polietileno y masillas de silicona 0 polisulfuros, dejando una cámara de descompresión detrás de dicho sellado.

Todas las operaciones descritas están sujetas a un control de calidad sistemático. Se realiza un control de todos los componentes del GRC y dos veces al día se controla el caudal de mortero y de fibras en la proyección del GRC. Se controla también el contenido de fibra y la relación agua/ materias sólidas en el GRC sin curar de acuerdo a lo establecido en la norma británica BS 6432-1984. A partir de muestras extraidas de paneles fabricados en idénticas condiciones a los reales, se llevan a cabo ensayos de flexión, de acuerdo a la norma anterior, con el fin de conocer el límite de proporcionalidad y el modulo de rotura del material. En algún caso particular se ensayan también a flexión paneles a escala real.

\section{APLICACIONES DEL GRC EN EDIFICIOS CONSTRUIDOS EN ARGELIA}

\subsection{Tipos de obras realizadas}

Como consecuencia del terremoto de El Asnam, del año 1980, el gobierno argelino necesitó de manera urgente reconstruir la ciudad de más de
200.000 habitantes equipándola de viviendas, colegios, hospitales y de todos los servicios complementarios. Los responsables argelinos vieron en los sistemas prefabricados e industrializados una salida a la urgencia con la que debian acometer las obras. Dragados y Construcciones propuso la ejecución de los edificios mediante una prefabricación de todos sus elementos, a base de estructuras metálicas atornilladas y forjados formados por losas de hormigón unidas a bastidores metálicos. Todas las fachadas se construyeron con paneles sandwich de GRC que daban una imagen de solidez y permitian una libertad de diseño no posible con otras soluciones a base de chapas metálicas u otros materiales.

El empleo del GRC permitió reducir los pesos de las piezas a menos de un tercio de lo que hubieran pesado las prefabricadas en hormigón, con el consiguiente importante ahorro en las operaciones de manipulación y transporte de las piezas asi como la simplificación de los sistemas de anclaje a la estructura de los edificios.

Este proyecto supuso la construcción de $290.000 \mathrm{~m}^{2}$ de edificios escolares, centros de salud y hospitales distribuidos en más de 80 ubicaciones diferentes de la misma zona.

Como consecuencia de la introducción de esta solución constructiva en el mercado argelino se consiguieron posteriormente otros contratos en las zonas de Argel, Tiaret y Mascara lo que su-

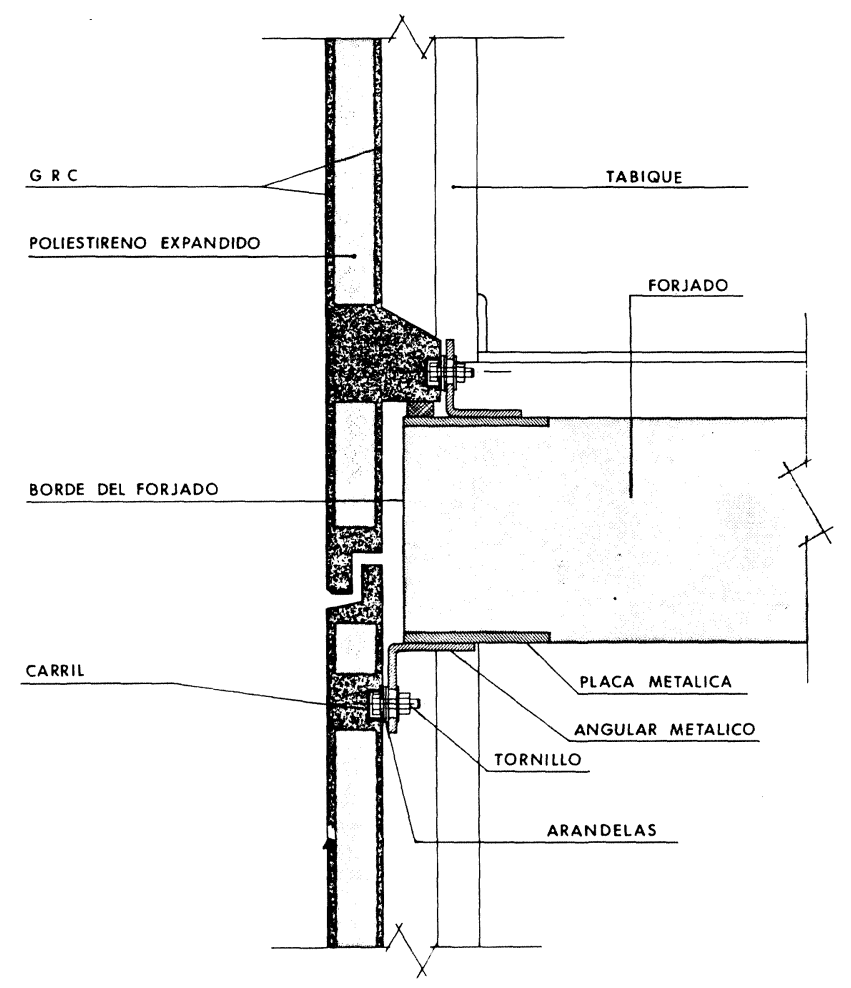

Sistema de anclaje de los paneles de GRC 
puso la construcción de $315.000 \mathrm{~m}^{2}$ de edificios de viviendas, edificios escolares y hospitales. El, conjunto de proyectos asi construidos en Argelia requirió fabricar, transportar y colocar 500.000 $\mathrm{m}^{2}$ de prefabricados de GRC en un plazo no superior a dos años, lo que fue posible gracias al empleo de este tipo de fachadas.

\subsection{Proyecto y construcción de los edificios}

El volumen de fachadas a prefabricar en GRC exigió una importante coordinación entre las tareas de proyecto, fabricación, transporte y montaje ya que se llegaron a obtener rendimientos punta de 120 paneles por dia.

Las fachadas se modularon en base a paneles de 2,7 m de ancho y 3-3,4 m de alto. Los paneles, del tipo sandwich, tenian $100 \mathrm{~mm}$ de espesor total con cáscaras de $10 \mathrm{~mm}$ conectadas entre si mediante nervios de GRC de $20 \mathrm{~mm}$, de espesor situados cada $0,6 \mathrm{~m}$, aproximadamente (Fig. 1). En los diseños iniciales el núcleo de los paneles estaba formado por fibra de vidrio, según lo especificado en el proyecto, lo que conlle-

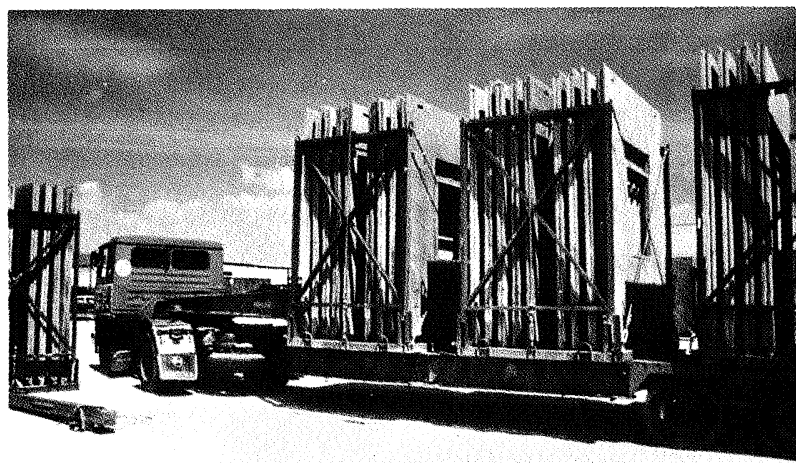

Fig. 4.

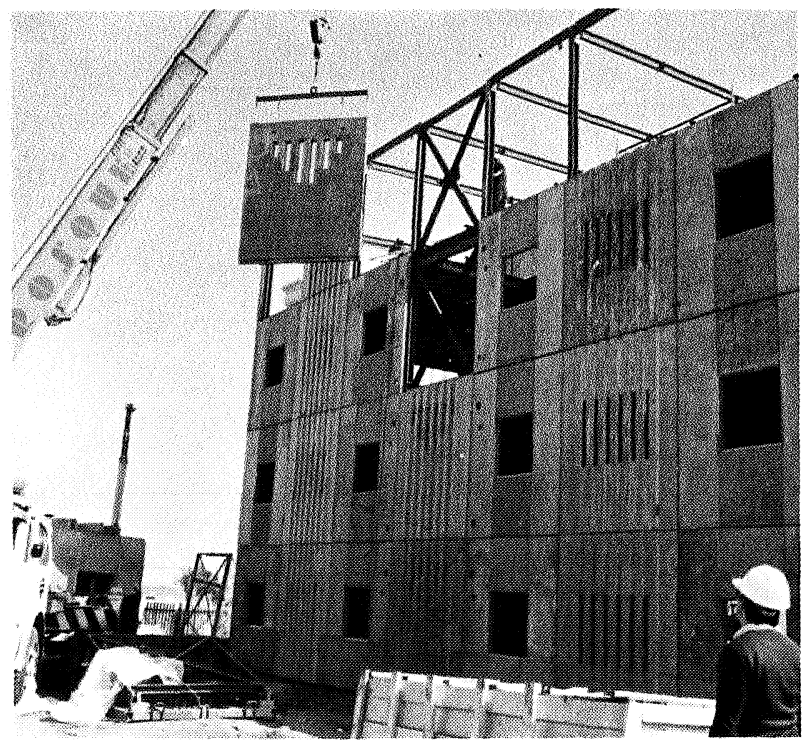

Fig. 6. vó algunos problemas en la terminación de su cara interior. Posteriormente los núcleos se construyeron con poliestireno expandido autoextinguible de $12 \mathrm{~kg} / \mathrm{m}^{3}$ de densidad mejorándose notablemente la calidad de los acabados de la cara interior de los paneles.

La fabricación de los paneles se realizó por el sistema de proyección en las factorias españolas de Madrid y Sevilla. Estos tenian incorporadas las carpinterias metálicas de aluminio y el acristalamiento de las puertas y las ventanas que era necesario proteger durante la proyección del GRC mediante maderas y aplicando al aluminio una laca pelable. La combinación de tratamientos superficiales pétreos y diversos tipos de pinturas conformaron la terminación de cada tipo de edificio.

Definidos los paneles en orden a optimizar el diseño, fabricación y transporte, fue necesario estudiar el sistema de envio, de forma que facilitase tanto el transporte como su posterior montaje en obra. Para ello, y desde la fase de proyecto, se definió la secuencia de montaje y a partir de ella la forma más racional de envio, para lo cual

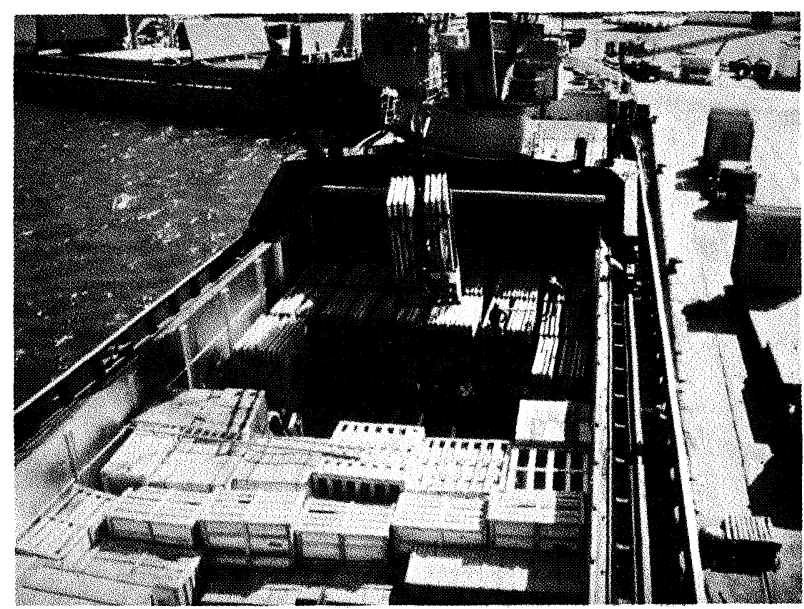

Fig. 5.

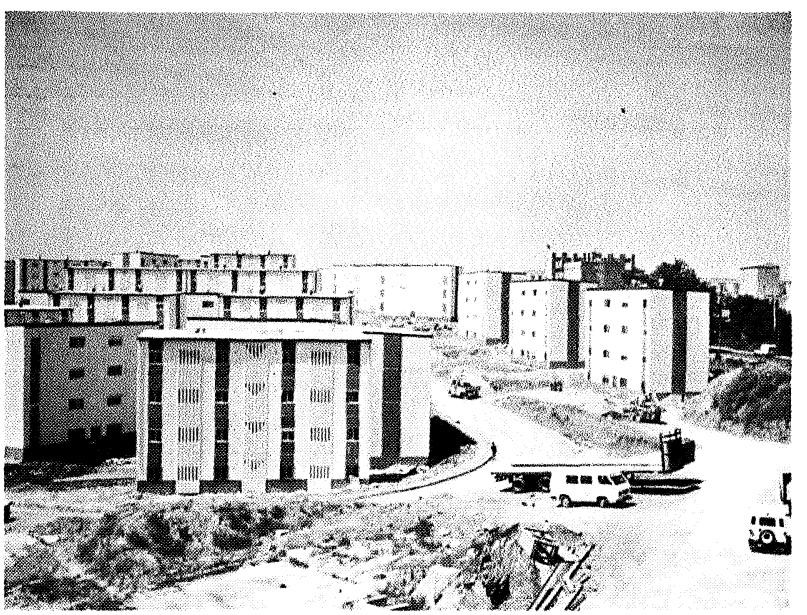

Fig. 7. 
de factoria salian expedidos los paneles agrupados en contenedores metálicos plegables. Estos se transportaban en camiones y barcos hasta su punto de destino (Fig. 4 a 7 ) de forma que llegaban directamente a pie de tajo, colocándolos en la posición adecuada para reducir al mínimo el movimiento de las grúas y poder construir las fachadas en el orden previsto. Los contenedores metálicos plegados volvian a sus puntos de destino para ser reutilizados en nuevos envios de paneles.

La dificultad de reponer los paneles defectuosos obligó a llevar un cuidadoso control de fabricación y manipulación de ellos y, en todo caso, al desarrollo de un sistema de reparación en obra de los pequeños daños. Se establecieron criterios para la aceptación o el rechazo de los paneles defectuosos. Los aceptados eran reparados con resina epoxi o con una premezcla de mortero de cemento con fibra de vidrio de $12 \mathrm{~mm}$ de longitud según el tipo de daño a reparar. Sin embargo, la necesidad de rechazar algunos paneles influyó en la decisión de montar en Argelia (Ech Cheliff) una pequeña factoria para la reposición de las piezas defectuosas que de otra manera obligaba a esperar un nuevo envío de los edificios.

obligaba a esperar un nuevo envio desde España con la consiguiente pérdida del ritmo de acabado de los edificios.

\section{APLICACIONES DEL GRC EN OBRAS CONSTRUIDAS EN ESPAÑA}

El empleo del GRC en España ha ido en aumento a lo largo de los últimos 10 años, utilizándose fundamentalmente en la construcción de fachadas como alternativa a soluciones de alta calidad.

En menor medida se ha utilizado en elementos de decoración interior y en la rehabilitación de fachadas de edificios ya construidos. También se ha utilizado en algunas aplicaciones especiales como cabinas, cubiertas de pasarelas, tumbas, encofrados perdidos de losas de hormigón, etc.

\subsection{Fachadas}

Las fachadas de GRC se han construido, en la mayor parte de los casos, con paneles sandwich con núcleos de poliestireno expandido. A título de ejemplo se mencionan a continuación algunas de las realizaciones construidas.

La figura 8 muestra un edificio de oficinas y viviendas de 24 plantas situado en una importante plaza de la capital de España. Las fachadas se construyeron con paneles sandwich de GRC de color rojizo conseguido por medio de la adición de un pigmento a la masa, y se terminaron con la aplicación de un barniz incoloro. Su núcleo estaba formado por una capa de hormigón con perlas de poliestireno y otra capa de poliestireno expandido. La figura 9 representa el edificio de la Delegación de Hacienda de Córdoba, cuyas fachadas se fabricaron en GRC con cemento blanco y los partesoles con cáscaras de GRC y núcleos de hormigón con perlas de poliestireno expandido.

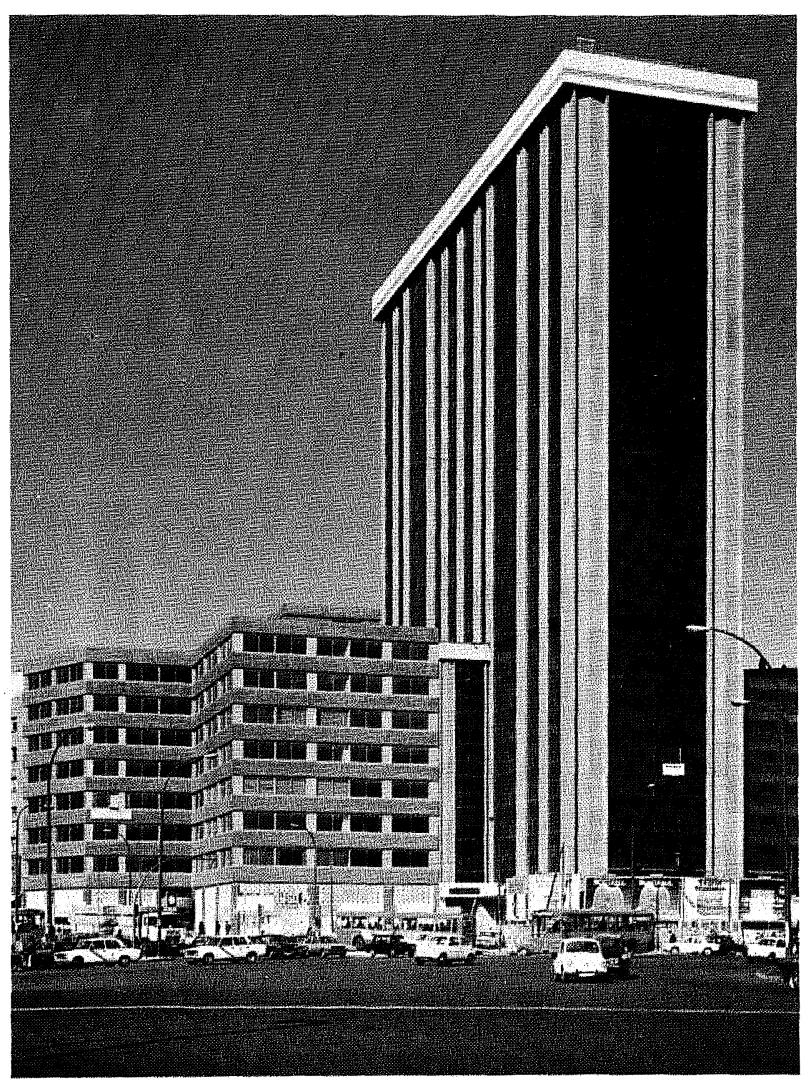

Fig. 8.

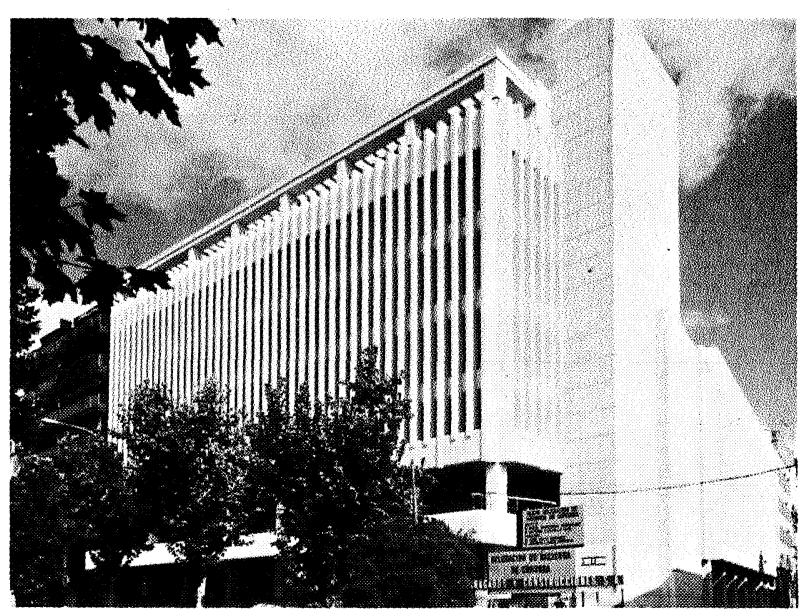

Fig. 9 
La figura 10 muestra un edificio recientemente construido para la sede de la Televisión gallega, situado en las proximidades de Santiago de Compostela. Fue necesario diseñar el edificio con soluciones que pudieran construirse en un plazo de nueve meses. El empleo del GRC en la construcción de todas las fachadas contribuyó a poder terminar este edificio de casi $7.000 \mathrm{~m}^{2}$ en el plazo requerido.

El siguiente edificio mostrado en la figura $11 \mathrm{co}-$ rresponde a un centro de seguimiento de satélites de la Compañía Telefónica Nacional ubicado en la provincia de Guadalajara. Sus $4.000 \mathrm{~m}^{2}$ de complicadas fachadas pudieron construirse gracias al empleo de paneles cáscara y paneles sandwich de GRC (Fig. 2). Los paneles cáscara tenian $10 \mathrm{~mm}$ de espesor con nervios de $20 \mathrm{~mm}$ de espesor y un canto total de $100 \mathrm{~mm}$. Se emplearon principalmente en la construcción de los zócalos y las cornisas. Los paneles sandwich tenian un canto total de $100 \mathrm{~mm}$ con cáscaras de $10 \mathrm{~mm}$, nervios de GRC de $20 \mathrm{~mm}$ de espesor y núcleos de poliestireno expandido y se emplearon en la construcción del cerramiento de las partes habitadas del edificio. La terminación de todos los paneles se hizo utilizando árido imitando la piedra de Segovia.

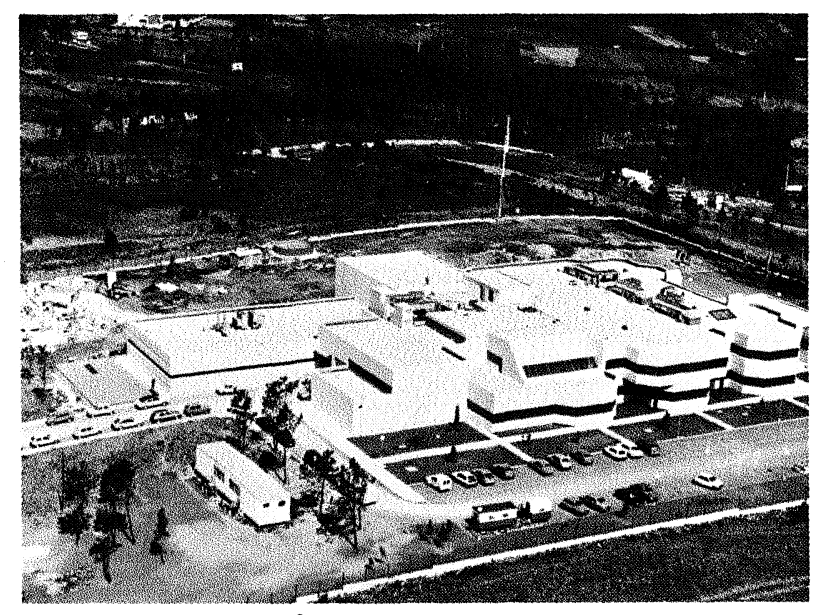

Fig. 10.

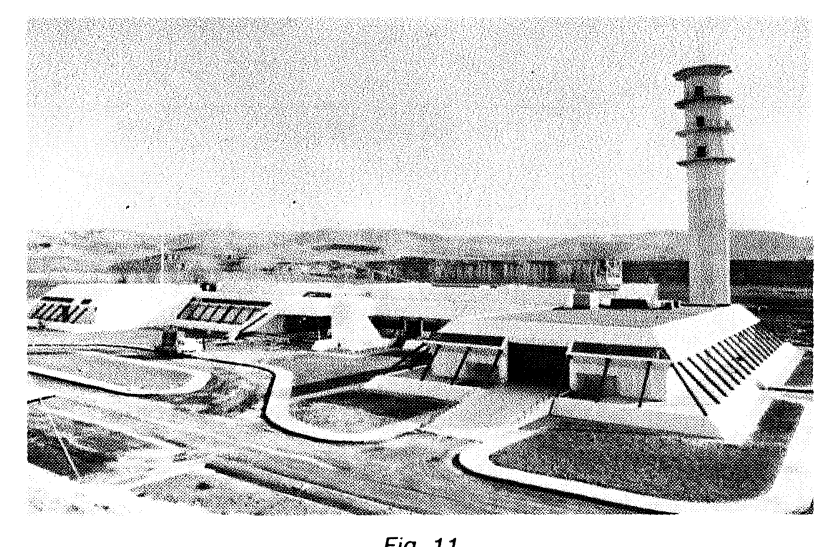

Fig. 11.

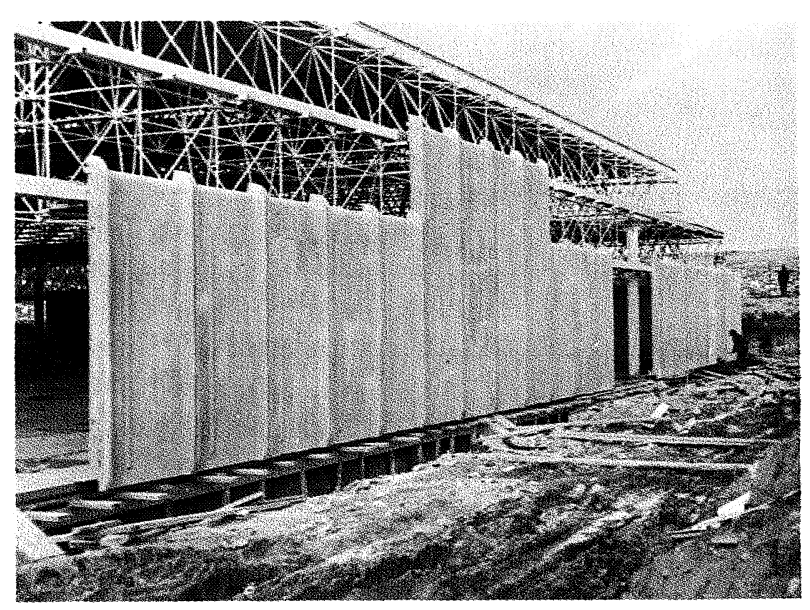

Fig. 12.

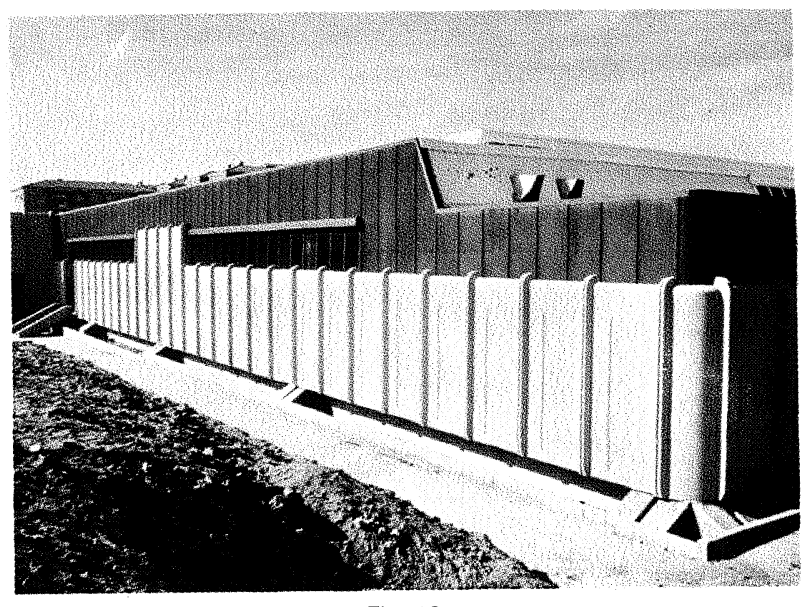

Fig. 13.

Las figuras 12 y 13 muestran un mercado en la provincia de Salamanca construido con una estructura metálica y cerramientos de GRC. Los paneles sandwich de 140 y $200 \mathrm{~mm}$ de canto tenian alturas variables entre 3,0 y $8,0 \mathrm{~m}$ y su terminación se realizó con árido calizo imitando la piedra de Salamanca (Valmayor).

Como último ejemplo del uso del GRC en fachadas de edificios se muestra en las figuras 14 y 15 las recientemente acabadas instalaciones para la sede central de los campeonatos mundiales de natación que se celebrarán el próximo mes de agosto en Madrid. La fachada se ha resuelto combinando el ladrillo con elementos sandwich de GRC acabados con una pintura de poliuretano. Los paneles tienen dimensiones múltiplo de 1,25 fabricándose y montándose paneles de hasta $3,75 \times 2,50 \mathrm{~m}$ con un espesor total de $100 \mathrm{~mm}$ que se anclaron a una estructura auxiliar de tubos de acero. 


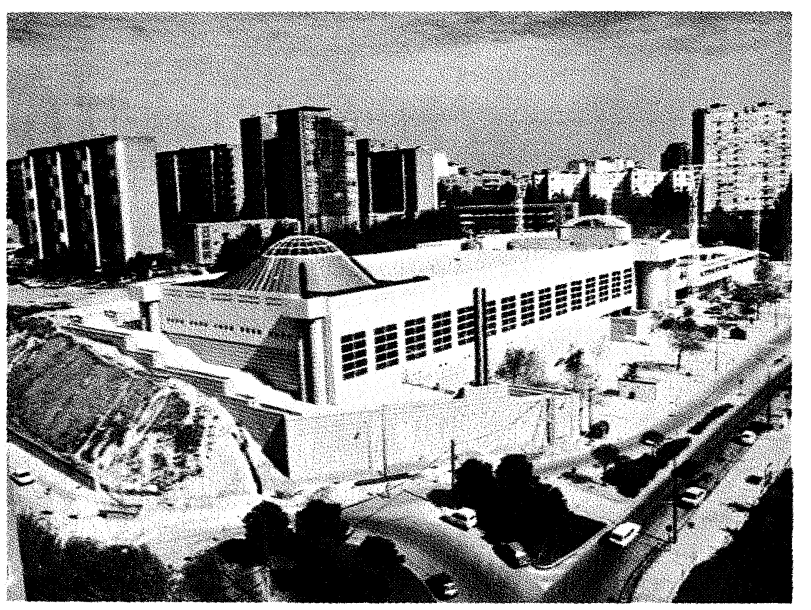

Fig. 14.

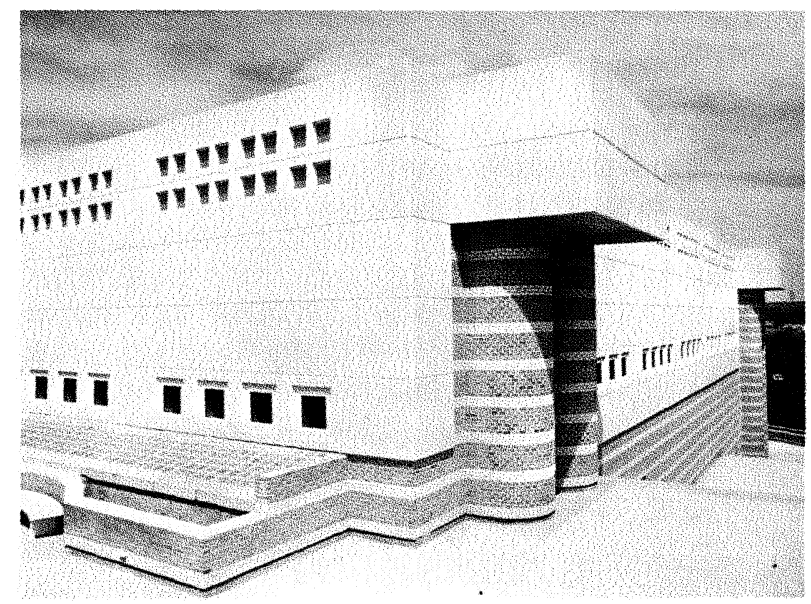

Fig. 15.

\subsection{Decoración interior}

En algunas obras especiales como la ya citada sede de los mundiales de natación se han construido elementos de GRC para el forrado de los pilares y para diversos detalles interiores, sin otra finalidad que la de obtener un determinado acabado interior de los edificios. Este tipo de solución también se ha empleado en la remodelación de la terminal nacional del aeropuerto de Barajas (Madrid) y en el aeropuerto de Valencia.

Las figuras 16 y 17 muestran la utilización del GRC como elemento de decoración del techo del nuevo vestíbulo de la estación de metro de la Puerta del Sol, en Madrid. La necesidad de tener que construir una losa de hormigón armado en el techo de dicho vestíbulo, que debe resistir el paso de tráfico rodado sobre ella, con un acabado inferior imitando un complicado artesonado, ha aconsejado la utilización del GRC como ele-

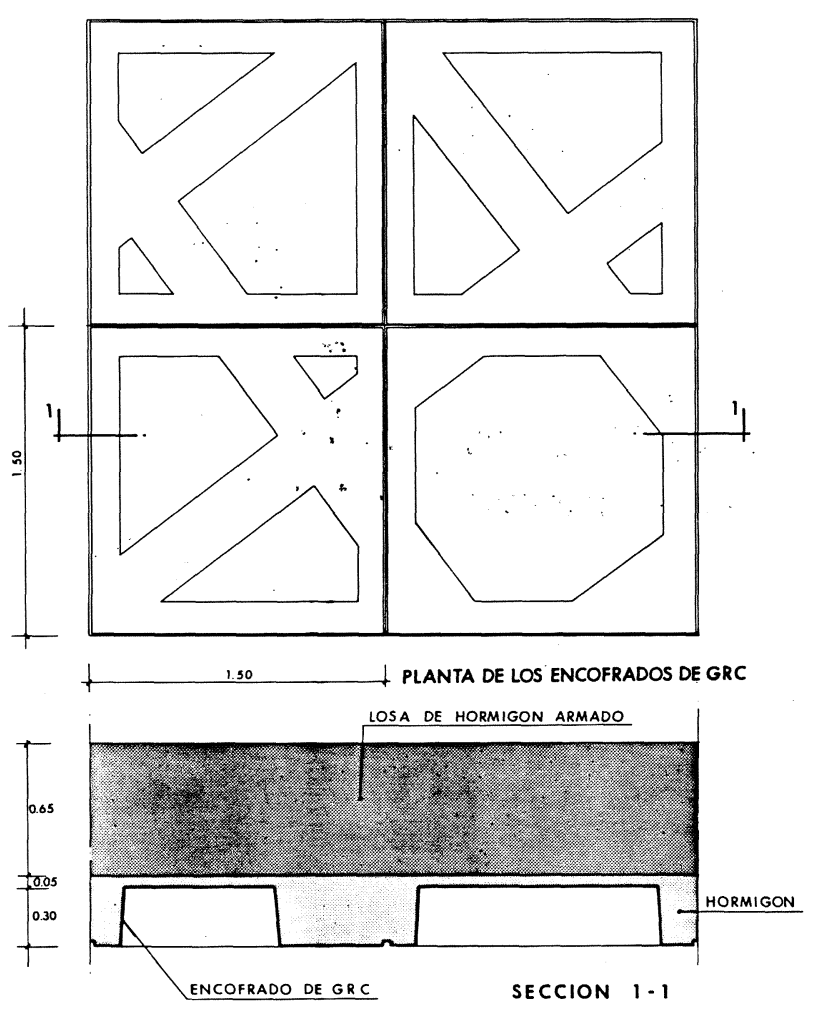

Esquema de la losa del techo del hall de la estación de metro "Puerta del Sol" (Madrid).

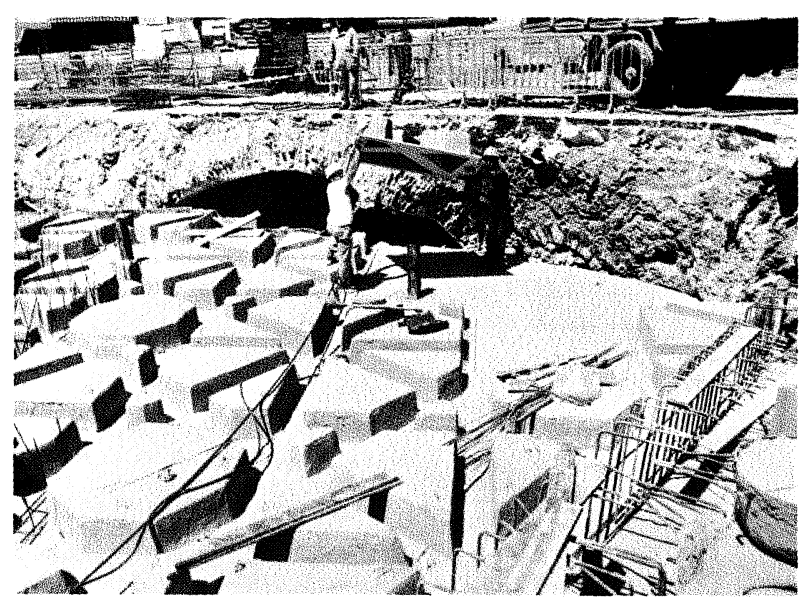

Fig. 16.

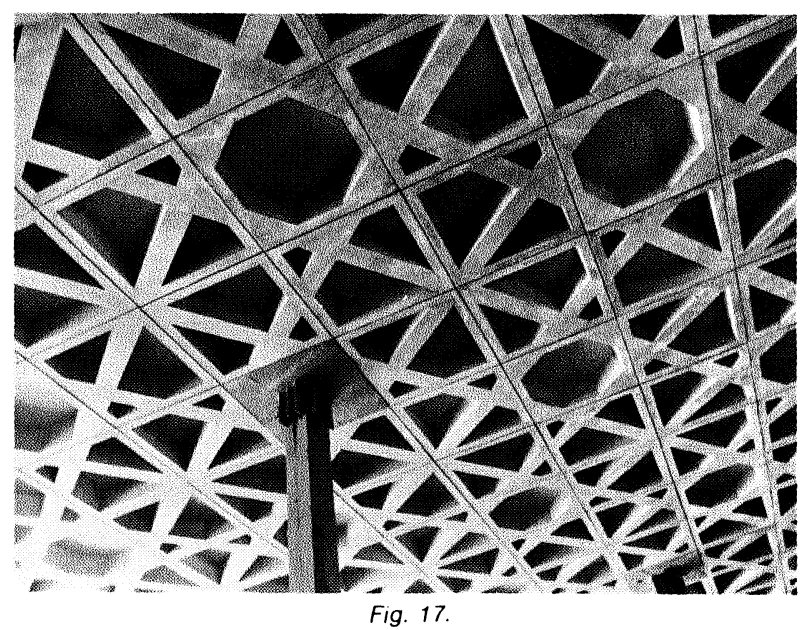


mento de terminación y simultáneamente como encofrado perdido. Las piezas de GRC son de $1,5 \times 1,5 \mathrm{~m}$, con $10 \mathrm{~mm}$ de espesor y una altura de $0,30 \mathrm{~m}$ equivalente a la altura de los nervios de la losa de hormigón (ver esquema pág. anterior). La construcción de ésta se realiza en 2 fases. En la primera, una vez colocadas las piezas de GRC, se hormigona la losa hasta una altura de 0,05 m por encima de la cara superior de las piezas de GRC. Cuando el hormigón ha alcanzado la resistencia requerida se prosigue el hormigonado del resto de la losa hasta completar el canto total de $1 \mathrm{~m}$. La obra se completa con el forrado de GRC de los pilares y los capiteles metálicos.

\subsection{Rehabilitación de fachadas y otras aplicaciones}

En menor medida, el material GRC está siendo utilizado en la rehabilitación de las fachadas de edificios ya construidos, que por cambio de uso o por defectos de las fachadas ha sido preciso reconstruir. La mayor dificultad del empleo del GRC en este campo estriba en su poca competitividad frente a otras soluciones alternativas más baratas, aunque de inferior calidad. En la parte derecha de la figura 18 se muestra la fachada rehabilitada con paneles sandwich de GRC de un edificio del poligono Santana de Madrid, mientras que en la parte izquierda de la misma figura puede observarse otro edificio del mismo tipo que aún no ha sido reparado.

El GRC se ha aplicado también a casos particulares como las cubiertas de los fingers de acceso a los aviones del aeropuerto de Madrid (Fig.

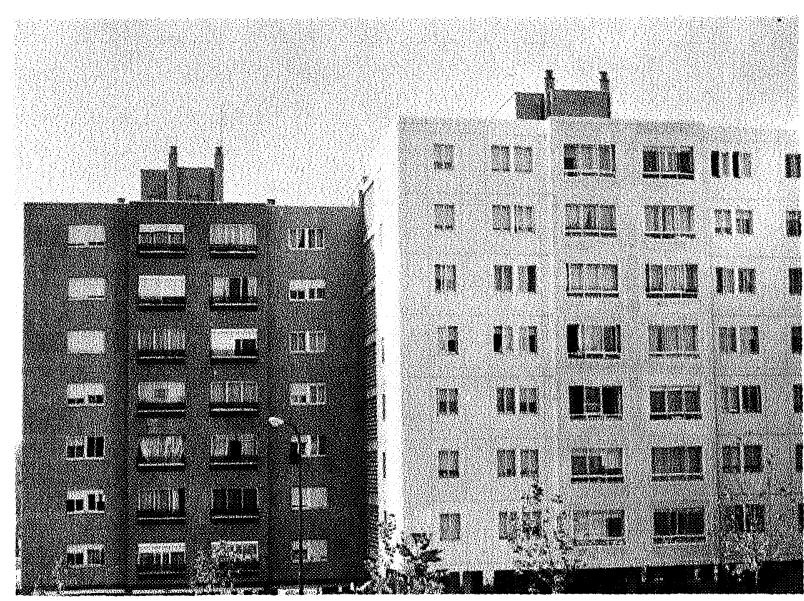

Fig. 18.

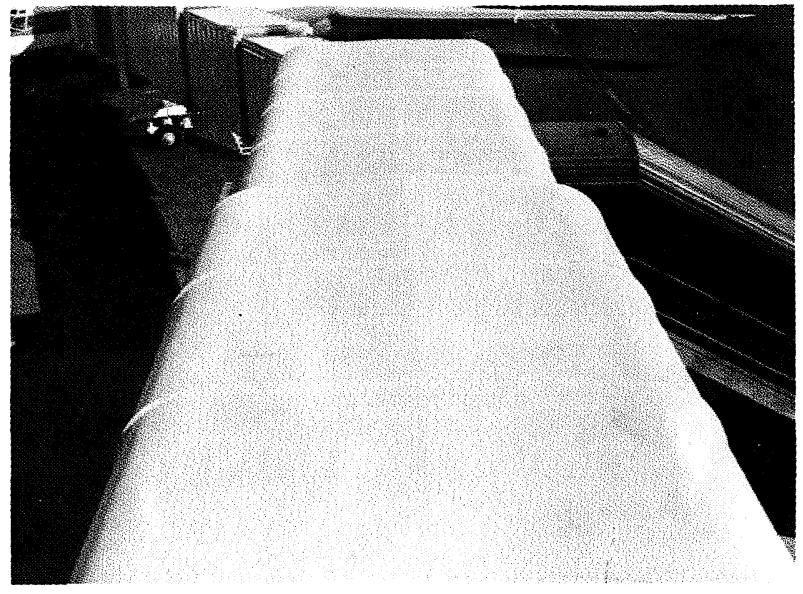

Fig. 19.

19) y las cubiertas y los cerramientos laterales de los del aeropuerto de Valencia.

También se han construido con este material tumbas en el cementerio de Burgos, centros rurales para Icona, etc.

Actualmente se están proyectando cabinas construidas totalmente en GRC, con dimensiones en planta de hasta 3,0 × 3,0 para alojar en su interior aparatos de mediciones hidráulicas en las cuencas de los rios, o para alojar los aparatos de los sistemas de comunicación tren-tierra de Renfe, etc. y en general para todo uso que prime el prefabricar elementos ligeros de fácil montaje en zonas rurales o montañosas de dificil acceso.

\section{CONSIDERACIONES FINALES}

El empleo del material GRC ha hecho posible la construcción de un gran número de fachadas en España y ha permitido llevar a cabo unos importantes proyectos en Argelia en un tiempo récord.

Actualmente se trabaja en el desarrollo de nuevos diseños de grandes paneles para fachadas, combinando perfiles metálicos muy ligeros de fabricación nacional con cáscaras de GRC.

Sin embargo, la profundización en el conocimiento del comportamiento a largo plazo del GRC (con fibras resistentes a los alcalis del cemento, o con polimeros añadidos a la mezcla) es el tema de mayor interés, que puede contribuir a incrementar el empleo de este material en la construcción. 